\title{
Analytical and approximate solution of two-dimensional convection-diffusion problems
}

\author{
Hatıra Günerhan* \\ Department of Mathematics, Faculty of Education, Kafkas University, Kars, Turkey \\ gunerhanhatira@gmail.com
}

\section{ARTICLE INFO}

\section{Article History:}

Received 04 February 2019

Accepted 16 May 2019

Available 14 January 2020

\section{Keywords:}

Reduced differential transform

method (RDTM)

Nonhomogeneous convection-diffusion equation

Two-dimensional convection-diffusion equation

AMS Classification 2010:

35R01; $76 R x x$

\begin{abstract}
In this work, we have used reduced differential transform method (RDTM) to compute an approximate solution of the Two-Dimensional ConvectionDiffusion equations (TDCDE). This method provides the solution quickly in the form of a convergent series. Also, by using RDTM the approximate solution of two-dimensional convection-diffusion equation is obtained. Further, we have computed exact solution of non-homogeneous CDE by using the same method. To the best of my knowledge, the research work carried out in the present paper has not been done, and is new. Examples are provided to support our work.
\end{abstract}

\section{Introduction}

We consider two-dimensional convection-diffusion equation as follows:

$$
\begin{aligned}
& \frac{\partial u(a, b, t)}{\partial t}+\beta_{a} \frac{\partial u(a, b, t)}{\partial a}+\beta_{b} \frac{\partial u(a, b, t)}{\partial b} \\
& =\alpha_{a} \frac{\partial^{2} u(a, b, t)}{\partial a^{2}}+\alpha_{b} \frac{\partial^{2} u(a, b, t)}{\partial b^{2}}+f(a, b, t), \\
& \text { in } \Omega \times(0, T], u(a, b, t)=g(a, b, t), \\
& (a, b) \in \partial \Omega, \quad \mathrm{t} \in(0, T], u(a, b, 0)=h(a, b), \\
& (a, b) \in \Omega,
\end{aligned}
$$

where $\beta_{a}$ and $\beta_{b}$ are progressive velocity components in the direction of $a$ and $b$ respectively, and $\alpha_{a}>0$ and $\alpha_{b}>0$ are the coefficients of diffusivity in the $a$ and $b$ directions, respectively. And $\alpha_{a}>0$ and $\alpha_{b}>0$ are $g(a, b, c)$ and $h(a, b)$ are smooth functions and $\Omega$ is a subset of $R^{2}$ and $(0, T]$ is the time interval, and $\partial \Omega$ is the boundary of $\Omega$.

This equation is frequently used in applied sciences and engineering especially in modeling and simulations of various complex phenomena in science and engineering. This paper first describes RDTM and then uses it to solve the Convectiondiffusion equation. In recent years, studies conducted on findings new analytical solutions of differential equations have attracted attention of scientists from all over the world (see [1]- [9]).

And some numerical solutions have been developed to solve these types of convection-diffusion problems. likes: Higher-Order ADI method [10] or rational high-order compact ADI method [11, the alternating direction implicit method [12], the finite element method [13, fourth-order compact finite difference method [14, decomposition Method [15], the finite difference method [16], restrictive taylors approximation [17, The fundamental solution [18], finite difference method [19], combined compact difference scheme and alternating direction implicit method [20, higher order compact schemes method [21, the finite volume method [22, the finite difference and legendre spectral method [23] and even the Monte

*Corresponding Author 
Carlo method [24]. Keskin in [25] proposed the RDTM to solve various PDE and fractional nonlinear partial differential equations.

This method is a repetitive procedure for the solution of a Taylor series differential equations. This technique reduces the size of the computational work and can be easily applied to numerous physical problems. We organize the paper as follows. In section RDTM is used to four types of TDCDP, and section 4 concludes the paper.

\section{Analysis of the RDTM}

We have a function with three variables $u(a, b, t)$, and presume that it can be shown as an invention of multiple of two functions $u(a, b, t)=$ $v(a, b) w(t) . u(a, b, t)$ can be denoted as

$$
\begin{aligned}
& u(a, b, t)= \\
& \left(\sum_{n_{1}=0}^{\infty} \sum_{n_{2}=0}^{\infty} V\left(n_{1}, n_{2}\right) a^{n_{1}} b^{n_{2}}\right) \cdot\left(\sum_{n_{3}=0}^{\infty} W\left(n_{3}\right) t^{n_{3}}\right) \\
& =\sum_{n_{1}=0}^{\infty} \sum_{n_{2}=0}^{\infty} \sum_{n_{3}=0}^{\infty} V\left(n_{1}, n_{2}\right) W\left(n_{3}\right) a^{n_{1}} b^{n_{2}} t^{n_{3}} \\
& =\sum_{k=0}^{\infty} U_{k}(a, b) t^{k},
\end{aligned}
$$

where $U_{k}(a, b)$ is called $t$-dimensional spectrum function of $u(a, b, t)$. The three-dimensional RDTM are introduced are defined in [26] as follows:

Definition 1. Assume $u(a, b, t)$ is an analytic function in the domain of interest. The RDTM of $u(a, b, t)$ is defined as

$$
U_{k}(a, b)=\frac{1}{k !}\left[\frac{\partial^{k}}{\partial t^{k}} u(a, b, t)\right]_{t=0} .
$$

Definition 2. The differential inverse transform of $U_{k}(a, b)$ is defined as:

$$
u(a, b, t)=\sum_{k=0}^{\infty} U_{k}(a, b) t^{k} .
$$

By inserting equation (3) in (4), we obtain

$$
u(a, b, t)=\sum_{k=0}^{\infty} \frac{1}{k !}\left[\frac{\partial^{k}}{\partial t^{k}} u(a, b, t)\right]_{\mathrm{t}=0} t^{k}
$$

Some basic properties of RDTM are presented in Table1 below.
Table 1. The operations for the reduced differential transform method.

\begin{tabular}{|l|l|}
\hline Original function & Transformed function \\
\hline$g(a, b, t) \pm h(a, b, t)$ & $G_{k}(a, b) \pm H_{k}(a, b)$ \\
\hline$e^{\gamma t}$ & $\frac{\gamma^{k}}{k !}$ \\
\hline$\frac{\partial^{c}}{\partial t^{c}} g(a, b, t)$ & $\frac{(k+c) !}{K !} G_{k+c}(a, b)$ \\
\hline$g(a, b, t) h(a, b, t)$ & $\sum_{l=0}^{k} G_{l}(a, b) H_{k-l}(a, b)$ \\
\hline$\frac{\partial^{w}}{\partial a^{w}} g(a, b, t)$ & $\frac{\partial^{w}}{\partial a^{w}} G_{k}(a, b)$ \\
\hline$a^{w} b^{v} t^{c}$ & $a^{w} b^{v} \delta(k-c)=\left\{\begin{array}{c}a^{w} b^{v}, k=c \\
0, k \neq c\end{array}\right.$ \\
\hline$\frac{\partial^{w+v+c}}{\partial a^{w} \partial b^{v} \partial t^{c}} g(a, b, t)$ & $\frac{\partial^{w+v}}{\partial a^{w} \partial b^{v}} \frac{(k+c) !}{k !} G_{k+c}(a, b)$ \\
\hline
\end{tabular}

\section{Applications}

We used the basic definitions (in Section 2) of the three-dimensional RDTM for solving four examples of Convection-diffusion equations (CDE).

Example 1. Consider the TDCDP (see [15])

$$
\frac{\partial u}{\partial t}-\frac{\partial^{2} u}{\partial a^{2}}-\frac{\partial^{2} u}{\partial b^{2}}=0,(a, b, t) \in \Omega \times J,
$$

with the initial condition

$$
u(a, b, 0)=\sin (\pi a) \sin (\pi b) .
$$

By using the RDTM in equations (6) and (7), we obtain

$(k+1) U_{k+1}(a, b)-\frac{\partial^{2}}{\partial a^{2}} U_{k}(a, b)-\frac{\partial^{2}}{\partial b^{2}} U_{k}(a, b)=0$,

from initial condition(7), we have

$$
U_{0}(a, b)=\sin (\pi a) \sin (\pi b) .
$$

By using Eq. (9) in Eq. (8), we obtain $U_{k}(a, b)$ values for $k=\{0,1,2,3, \cdots\}$ as follows:

$$
\begin{aligned}
& U_{1}(a, b)=-2 \pi^{2} \sin (\pi a) \sin (\pi b) \\
& U_{2}(a, b)=2 \pi^{4} \sin (\pi a) \sin (\pi b) \\
& U_{3}(a, b)=-\frac{4}{3} \pi^{6} \sin (\pi a) \sin (\pi b) \\
& U_{4}(a, b)=\frac{2}{3} \pi^{8} \sin (\pi a) \sin (\pi b) \\
& U_{5}(a, b)=-\frac{4}{15} \pi^{10} \sin (\pi a) \sin (\pi b) \\
& U_{6}(a, b)=\frac{4}{45} \pi^{12} \sin (\pi a) \sin (\pi b) \\
& U_{7}(p, q)=-\frac{8}{315} \pi^{14} \sin (\pi a) \sin (\pi b), \ldots
\end{aligned}
$$

by using the differential inverse reduced transform of $U_{k}(a, b)$,we get 


$$
\begin{aligned}
& u(a, b, t)=\sum_{k=0}^{\infty} U_{k}(a, b) t^{k} \\
& \quad=U_{0}(a, b)+U_{1}(a, b) t+U_{2}(a, b) t^{2}+\ldots \\
& \quad=\sin (\pi a) \sin (\pi b)\left(1-2 \pi^{2} t+2 \pi^{4} t^{2}-\frac{4}{3} \pi^{6} t^{3}\right. \\
& \left.\quad+\frac{2}{3} \pi^{8} t^{4}-\frac{4}{15} \pi^{10} t^{5}+\frac{4}{45} \pi^{12} t^{6}+\ldots\right),
\end{aligned}
$$

by using the closed form in the solution of (11), we obtain following approximate solution

$$
u(a, b, t)=\sin (\pi a) \sin (\pi b) e^{-2 \pi^{2} t} .
$$

Example 2. We consider the non-homogeneous convection-diffusion problem see ( [15])

$\frac{\partial u}{\partial t}+\frac{\partial u}{\partial a}+\frac{\partial u}{\partial b}-\frac{\partial^{2} u}{\partial a^{2}}-\frac{\partial^{2} u}{\partial b^{2}}=3 a^{2}-6 a+2 t+1$

$$
(a, b, t) \in \Omega \times J
$$

subject to the initial condition

$$
u(a, b, 0)=a^{3}+b .
$$

By using the basic properties of RDTM in equations (13) and (14), we obtain the following relations

$$
\begin{aligned}
& (k+1) U_{k+1}(a, b)+\frac{\partial}{\partial a} U_{k}(a, b)+\frac{\partial}{\partial b} U_{k}(a, b) \\
& -\frac{\partial^{2}}{\partial a^{2}} U_{k}(a, b)-\frac{\partial^{2}}{\partial b^{2}} U_{k}(a, b) \\
& =3 a^{2} \delta(k)-6 a \delta(k)+2 \delta(k-1)+\delta(k)
\end{aligned}
$$

Taking the differential transform of Eq.(14), we write

$$
U_{0}(a, b)=a^{3}+b .
$$

By using Eq. (16) in Eq. (15), we obtain $U_{k}(a, b)$ values fork $=\{0,1,2,3, \cdots\}$ as follows

$$
\begin{aligned}
& U_{1}(a, b)=0, U_{2}(a, b)=1, U_{i}(a, b)=0, \\
& \operatorname{for}(i=3,4,5, \ldots) .
\end{aligned}
$$

The exact solution of the equation (13) will assume the following form:

$$
u(a, b, t)=\sum_{k=0}^{\infty} U_{k}(a, b) t^{k}=a^{3}+b+t .
$$

Example 3. We consider the non-homogeneous CDE (see [14])

$$
\frac{\partial u}{\partial t}-\frac{\partial^{2} u}{\partial a^{2}}-\frac{\partial^{2} u}{\partial b^{2}}+\frac{\partial u}{\partial a}=\left(2 \pi^{2}-1\right) e^{-t} \sin (\pi a) \cos (\pi b)
$$

$$
+\pi e^{-t} \cos (\pi a) \cos (\pi b), \quad(a, b, t) \in \Omega \times J,
$$

with the initial condition

$$
u(a, b, 0)=\sin (\pi a) \cos (\pi b) .
$$

By using the basic properties of RDTM in equations (19) and (20), we obtain the following relations

$$
\begin{aligned}
(k+1) & U_{k+1}(a, b)-\frac{\partial^{2}}{\partial a^{2}} U_{k}(a, b) \\
& -\frac{\partial^{2}}{\partial b^{2}} U_{k}(a, b)+\frac{\partial}{\partial a} U_{k}(a, b) \\
= & \left(2 \pi^{2}-1\right) \frac{(-1)^{k}}{k !} \sin (\pi a) \cos (\pi b) \\
+ & \pi \frac{(-1)^{k}}{k !} \cos (\pi a) \cos (\pi b),
\end{aligned}
$$

from initial condition(20), we have

$$
U_{0}(a, b)=\sin (\pi a) \cos (\pi b) .
$$

By using Eq. (22) in Eq. (21), we obtain $U_{k}(a, b)$ values for $k=\{0,1,2,3, \cdots\}$ as follows:

$$
\begin{aligned}
& U_{1}(a, b)=-\sin (\pi a) \cos (\pi b), \\
& U_{2}(a, b)=\frac{1}{2} \sin (\pi a) \cos (\pi b), \\
& U_{3}(a, b)=-\frac{1}{6} \sin (\pi a) \cos (\pi b), \\
& U_{4}(a, b)=\frac{1}{24} \sin (\pi a) \cos (\pi b), \\
& U_{5}(a, b)=-\frac{1}{120} \sin (\pi a) \cos (\pi b), \\
& U_{6}(a, b)=\frac{1}{720} \sin (\pi a) \cos (\pi b), \\
& U_{7}(a, b)=-\frac{1}{5040} \sin (\pi a) \cos (\pi b), \ldots,
\end{aligned}
$$

by using the differential inverse reduced transform of $U_{k}(a, b)$,we get

$$
\begin{aligned}
& u(a, b, t)=\sum_{k=0}^{\infty} U_{k}(a, b) t^{k}=U_{0}(a, b)+U_{1}(a, b) t+\ldots \\
& =\sin (\pi a) \cos (\pi b)\left(1-t+\frac{t^{2}}{2 !}-\frac{t^{3}}{3 !}+\frac{t^{4}}{4 !}-\frac{t^{5}}{5 !}+\frac{t^{6}}{6 !}-\ldots\right),
\end{aligned}
$$

by using the closed form in the solution of (24), we obtain the following exact solution

$$
u(a, b, t)=e^{-t} \sin (\pi a) \cos (\pi b) .
$$

Example 4. Consider the TDCDP (see [14])

$$
\begin{aligned}
& \frac{\partial u}{\partial t}=\frac{\partial^{2} u}{\partial a^{2}}+\frac{\partial^{2} u}{\partial b^{2}}-\frac{\partial u}{\partial a} \\
& +e^{-t}\left(2 \pi^{2}-1\right) \sin (\pi a) \sin (\pi b) \\
& +\pi e^{-t} \cos (\pi a) \sin (\pi b),(a, b, t) \in \Omega \times J
\end{aligned}
$$

with the initial condition

$$
u(a, b, 0)=\sin (\pi a) \sin (\pi b) .
$$


By using the basic properties of RDTM in equations (26) and (27), we obtain the following relations

$$
\begin{array}{r}
(k+1) U_{k+1}(a, b)=\frac{\partial^{2}}{\partial a^{2}} U_{k}(a, b)+\frac{\partial^{2}}{\partial b^{2}} U_{k}(a, b) \\
-\frac{\partial}{\partial a} U_{k}(a, b)+\left(2 \pi^{2}-1\right) \frac{(-1)^{k}}{k !} \sin (\pi a) \sin (\pi b) \\
+\pi \frac{(-1)^{k}}{k !} \cos (\pi a) \sin (\pi b), \quad(28
\end{array}
$$

from initial condition(28), we have

$$
U_{0}(a, b)=\sin (\pi a) \sin (\pi b) .
$$

By using Eq. (29) in Eq. (28), we obtain $U_{k}(a, b)$ values for $k=\{0,1,2,3, \cdots\}$

$$
\begin{aligned}
& U_{1}(a, b)=-\sin (\pi a) \sin (\pi b), \\
& U_{2}(a, b)=\frac{1}{2} \sin (\pi a) \sin (\pi b), \\
& U_{3}(a, b)=-\frac{1}{6} \sin (\pi a) \sin (\pi b), \\
& U_{4}(a, b)=\frac{1}{24} \sin (\pi a) \sin (\pi b), \\
& U_{5}(a, b)=-\frac{1}{120} \sin (\pi a) \sin (\pi b), \\
& U_{6}(a, b)=\frac{1}{720} \sin (\pi a) \sin (\pi b), \\
& U_{7}(a, b)=-\frac{1}{5040} \sin (\pi a) \sin (\pi b), \ldots,
\end{aligned}
$$

by using the differential inverse reduced transform of $U_{k}(a, b)$,we get

$$
\begin{aligned}
u(a, b, t) & =\sin (\pi a) \sin (\pi b)\left(1-t+\frac{t^{2}}{2}\right. \\
& \left.-\frac{t^{3}}{6}+\frac{t^{4}}{24}-\frac{t^{5}}{120}+\frac{t^{6}}{720}-\ldots\right),
\end{aligned}
$$

by using the closed form in the solution of (31) we obtain the following exact solution

$$
u(a, b, t)=e^{-t} \sin (\pi a) \sin (\pi b) .
$$

\section{Conclusion}

In this study, we used RDTM to solve convectiondiffusion problems and showed that RDTM is an effective and appropriate technique for finding exact solutions of the TDCDP which we have investigated here. On the other hand the results are quite reliable for solving this problem. The exact closed form solution was obtained for all the examples presented in this paper. RDTM offers an excellent opportunity for future research.

\section{References}

[1] Baskonus, H.M. (2019). Complex Soliton Solutions to the Gilson-Pickering Model. $A x$ ioms, 8(1), 18.
[2] Ilhan, O.A., Esen, A., Bulut, H., \& Baskonus, H.M. (2019). Singular Solitons in the Pseudoparabolic Model Arising in Nonlinear Surface Waves. Results in Physics, 12, 17121715.

[3] Cattani, C., Sulaiman, T.A., Baskonus, H.M., \& Bulut, H. (2018). Solitons in an inhomogeneous Murnaghan's rod. European Physical Journal Plus, 133(228), 1-12.

[4] Baskonus, H.M., Sulaiman, T.A. \& Bulut, H. (2018). Dark, bright and other optical solitons to the decoupled nonlinear Schrdinger equation arising in dual-core optical fibers. Optical and Quantum Electronics, 50(4), 1-12.

[5] Ciancio, A., Baskonus, H.M., Sulaiman, T.A., \& Bulut, H. (2018). New Structural Dynamics of Isolated Waves Via the Coupled Nonlinear Maccari's System with Complex Structure. Indian Journal of Physics, 92(10), 12811290.

[6] Ilhan, O.A., Sulaiman, T.A., Bulut, H., \& Baskonus, H.M. (2018). On the New Wave Solutions to a Nonlinear Model Arising in Plasma Physics. European Physical Journal Plus, 133(27), 1-6.

[7] Yel, G., Baskonus, H.M., \& Bulut, H. (2017). Novel archetypes of new coupled KonnoOono equation by using sineGordon expansion method. Optical and Quantum Electronics, 49(285), 1-10.

[8] Baskonus, H.M. (2017). New Complex and Hyperbolic Function Solutions to the Generalized Double Combined Sinh-Cosh-Gordon Equation. AIP Conference Proceedings. 1798, 1-9 (020018).

[9] Baskonus, H.M. (2016). New acoustic wave behaviors to the DaveyStewartson equation with power-law nonlinearity arising in fluid Dynamics. Nonlinear Dynamics, 86(1), 177183.

[10] Karaa, S., \& Zhang, J. (2004). Higher order ADI method for solving unsteady convectiondiffusion problems. Journal of Computational Physics, 198, 1-9.

[11] Tian, Z. (2011). A rational high-order compact ADI method for unsteady convectiondiffusion equations. Computer Physics Communications, 182, 649-662.

[12] Rui, H. (2003). An alternative direction iterative method with second-order upwind scheme for convection-diffusion equations. International Journal of Computer Mathematics, 80(4), 527-533.

[13] Mekuria, G.T., \& Rao, J.A. (2016). Adaptive finite element method for steady convectiondiffusion equation. American Journal of Computational Mathematics, 6(3), 275-285. 
[14] Li, L., Jiang, Z., \& Yin, Z. (2018). Fourthorder compact finite difference method for solving two-dimensional convection-diffusion equation. Advances in Difference Equations, 2018, 1-24.

[15] Momani, S. (2008). A Decomposition Method for Solving Unsteady ConvectionDiffusion Problems. Turkish Journal of Mathematics, 32, 51-60.

[16] Saqib, M., Hasnain, S., \& Mashat, D.S. (2017). Computational solutions of two dimensional convection-diffusion equation using Crank Nicolson and time efficient ADI. American Journal of Computational Mathematics, 7(3), 208-227.

[17] Ismail, H.N.A., Elbarbary, E. M. E., \& Salem, G. S. E. (2004). Restrictive Taylor's approximation for solving convectiondiffusion equation. Applied Mathematics and Computation, 147, 355-363.

[18] Castillo, M., \& Power, H. (2008). The Neumann series as a fundamental solution of the two-dimensional convection-diffusion equation with variable velocity. Journal of Engineering Mathematics, 62, 189-202.

[19] Noye, B.J., \& Tan, H.H. (1989). Finite difference methods for solving the twodimensional advection-diffusion equation. International Journal for Numerical Methods in Fluids, 9(1), 75-98.

[20] Sun, H., \& Li, L. (2014). A CCD-ADI method for unsteady convection-diffusion equations. Computer Physics Communications, 185, 790-797.

[21] Kalita, J.C., Dalal, D.C., \& Dass, A.K. (2002). A class of higher order compact schemes for the unsteady two-dimensional convection-diffusion equation with variable convection coefficients. International Journal for Numerical Methods in Fluids, 38, 11111131.

[22] Shu, C.W. (2017). Bound-preserving high order finite volume schemes for conservation laws and convection-diffusion equations. Finite Volumes for Complex Applications VIIIMethods and Theoretical Aspects, Springer Proceedings, 3-14.

[23] Ammi, M.R.S., \& Jamiai, I. (2017). Finite difference and Legendre spectral method for a time-fractional diffusion-convection equation for image restoration. Discrete $\&$ Continuous Dynamical Systems, 11(1), 103-117.

[24] Koley, U., Risebro, N.H., Schwab, C., et al. (2017). A multilevel monte carlo finite difference method for random scalar degenerate convection-diffusion equations. Journal of Hyperbolic Differential Equations, 14(3), 415454.

[25] Keskin, Y. (2010). Solving partial differential equations by the reduced differential transform method. PhD Thesis. Selcuk University.

[26] Ziqan, A.M., Armiti, S., \& Suwan, L. (2016). Solving three-dimensional volterra integral equation by the reduced differential transform method. International Journal of Applied Mathematical Research, 5(2), 103-106.

Hatıra Günerhan is currently an assistant professor at Kafkas University Faculty of Education, Department of Mathematics, Kars, Turkey. Her research interests are in the areas of applied mathematics including the numerical methods for special models of differential equations and differential-algebraic equations and integral equations.

(10) http://orcid.org/0000-0002-7802-477X

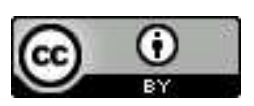

This work is licensed under a Creative Commons Attribution 4.0 International License. The authors retain ownership of the copyright for their article, but they allow anyone to download, reuse, reprint, modify, distribute, and/or copy articles in IJOCTA, so long as the original authors and source are credited. To see the complete license contents, please visit http://creativecommons.org/licenses/by/4.0/. 